\title{
On the Critical Separation Distance of Binary Vortices in a Nondivergent Barotropic Atmosphere
}

\author{
Seol-Eun SHIN ${ }^{1}$ \\ Department of Environmental Science and Engineering, Gwangju Institute of Science and Technology, \\ Gwangju, Korea
}

Ji-Young HAN and Jong-Jin BAIK

School of Earth and Environmental Sciences, Seoul National University, Seoul, Korea

(Manuscript received 14 January 2005, in final form 17 May 2006)

\begin{abstract}
The motion of binary cyclonic vortices with equal structure and strength in no environmental flow is investigated with a focus on what determines their merger or separation. For this, proposals in previous studies on the critical separation distance of binary vortices are validated using a nondivergent barotropic model on an $f$-plane.

Results from numerical experiments indicate that the sign of the initial relative vorticity in the midregion between two vortices largely determines their merger or separation. This is consistent with the proposal of Falkovich et al. When the initial relative vorticity in the mid-region between two vortices is negative, the two vortices separate. However, it is found that an initial positive relative vorticity between the two vortices does not guarantee their merger. Only when the initial positive relative vorticity exceeds a certain threshold value, the two vortices merge. Based upon the results of numerical experiments, it is suggested that the critical separation distance of binary vortices is slightly smaller than twice the radius at which the relative vorticity of one vortex becomes zero. The merger or separation of binary vortices can be conceptually explained by the advection of the symmetric relative vorticity of one vortex by the symmetric tangential flow of the other vortex. This relative vorticity advection produces vorticity anomalies with different magnitudes to the north and south of each vortex and results in net eastward or westward secondary flow near its center when the two vortices are initially placed in the east-west direction.
\end{abstract}

\section{Introduction}

Two tropical cyclones can attract or repel each other when they are close enough for mutual interaction. These tropical cyclones are called binary tropical cyclones. Dong and Neu-

Corresponding author: Jong-Jin Baik, School of Earth and Environmental Sciences, Seoul National University, Seoul 151-742, Korea. E-mail: jjbaik@snu.ac.kr

1 Present affiliation: Meteorological Institute, University of Munich, Munich, Germany. (C) 2006, Meteorological Society of Japan mann (1983) showed that the average frequency of binary tropical cyclones was three per two years over the western North Pacific and one per three years over the North Atlantic for the 36-year period 1946-1981. Lander (1995) and Kuo et al. (2000) analyzed merging binary tropical cyclones. Since the motion of binary tropical cyclones is more complex, track prediction errors for binary tropical cyclones can be much larger than those for a single tropical cyclone (Brand 1970). Chan and Lam (1989) and Chan (1995) gave actual examples of predicting the tracks of typhoons in mutual 
interaction. Therefore, an investigation of the core dynamics that complicates the motion of binary tropical cyclones is necessary to improve their track prediction.

Fujiwhara (1921, 1923, 1931) pioneered the investigation of how two vortices interact with each other by performing laboratory experiments and analyzing binary vortices in nature. He discovered that when two cyclonic vortices are located relatively close to each other, they rotate cyclonically around each other while the separation distance between them decreases with time. This is commonly referred to as the Fujiwhara effect. Haurwitz (1951) suggested that the rotational rate of two vortices is proportional to the intensity of circulation and inversely proportional to the square of separation distance.

Using a nondivergent barotropic model and a three-dimensional baroclinic model, Chang (1983) examined the interaction of two tropical cyclones. He argued that the divergent component of the winds rather than vorticity advection is responsible for the mutual attraction of two tropical cyclones on an $f$-plane. On the contrary, DeMaria and Chan (1984), using a nondivergent barotropic model, showed that two cyclonic vortices on an $f$-plane can also attract each other and eventually merge by relative vorticity advection only. They argued that the merger or separation of two vortices is determined by the sign of the relative vorticity gradient of one vortex at the center of the other vortex. Pokhil et al. (1990) proposed that the merger or separation of two vortices depends on the critical value of the ratio of the separation distance to the radius of the maximum wind averaged for the two vortices. Chan and Law (1995) focused on the advection of symmetric vorticity by asymmetric flow and the advection of asymmetric vorticity by symmetric flow in the mutual interaction of binary vortices. They pointed out that the steering as given by the advection of symmetric vorticity by asymmetric flow is not adequate to describe the motion of binary vortices and that the contribution from other vorticity advection components should be also considered. They also pointed out that the critical distance for merger to occur can be very different depending on the vortex structure. Falkovich et al. (1995) suggested that it is the sign of the relative vorticity be- tween two vortices that determines the merger or separation of binary vortices.

There have been several studies employing contour dynamics to examine the merger or separation of two vortices. Melander et al. (1988) suggested that the bifurcation behavior between merging and non-merging vortices results from a nonlinear interaction between the restoring capacity of each vortex and the strain imposed on each vortex by the shearing potential flow of the other vortex. On the basis of the study by Melander et al. (1988), Ritchie and Holland (1993) and Holland and Dietachmayer (1993) calculated the critical separation distance using the ratio of the strain to the restoring capacity and indicated that two vortices can merge when they are located within 150$300 \mathrm{~km}$. Their studies also indicate that the initial attraction of two vortex patches during the merging process results entirely from the changing vorticity advection as weak outer vorticity fields become distorted.

The interaction of binary tropical cyclones was also investigated using a coupled atmosphere-ocean model (Falkovich et al. 1995). Their results show that the impact of the tropical cyclone-ocean interaction on the motion is stronger for binary tropical cyclones than for a single tropical cyclone. The roles of background wind shear in the interaction between two vortices were studied using barotropic models (Chan and Law 1995; Hart and Evans 1999). Chan and Law (1995) showed that the environmental wind shear does not necessarily play the role of a steering flow, but it can change the pattern of various vorticity advection components. Hart and Evans (1999) showed that the interaction between $\beta$-gyre and background wind shear can change the motion of binary vortices.

The previous studies mentioned above have improved our understanding of the motion of binary tropical cyclones. However, there still remain many problems to be resolved. One of them is to find a reliable criterion that enables us to determine whether two tropical cyclones will merge or separate. This is particularly important since the tracks of two tropical cyclones can be substantially different from each other, depending on merger or separation. This study tries to answer this problem in the context of nondivergent barotropic vortex motion. In sec- 
tion 2, the numerical model used in this study is described. In section 3, previous ideas on the criterion determining the merger or separation of two vortices are reviewed and discussed. In section 4, results from numerical experiments on an $f$-plane are presented and discussed in terms of the critical separation distance and then our suggestion is made. Finally, a summary and conclusions are given in section 5 .

\section{Numerical model}

The numerical model developed for this study is a nondivergent barotropic model. The nondivergent barotropic vorticity equation is given by

$$
\frac{\partial \zeta}{\partial t}+u \frac{\partial \zeta}{\partial x}+v \frac{\partial \zeta}{\partial y}+\beta v=0,
$$

where $\zeta$ is the relative vorticity and $u$ and $v$ are the zonal and meridional velocity components, respectively. $\beta$ is the latitudinal gradient of the Coriolis parameter $f$. Since the flow is nondivergent, $\zeta, u$, and $v$ can be related to the streamfunction $\psi$ by the following equations.

$$
\begin{aligned}
& \zeta=\nabla^{2} \psi, \\
& u=-\frac{\partial \psi}{\partial y}, \quad v=\frac{\partial \psi}{\partial x} .
\end{aligned}
$$

Following DeMaria and Chan (1984), the initial axisymmetric vortex profile is given by

$$
V(r)=V_{m}\left(\frac{r}{r_{m}}\right) \exp \left\{\frac{1}{b}\left[1-\left(\frac{r}{r_{m}}\right)^{b}\right]\right\},
$$

where $r$ is the radius from the vortex center, $V(r)$ the tangential wind speed, $V_{m}$ the maximum tangential wind speed, and $r_{m}$ the radius of maximum wind. The factor $b$ in (4) determines the decay rate of the tangential wind with radius outside $r=r_{m}$. The initial relative vorticity structure corresponding to (4) is

$\zeta(r)=\frac{2 V_{m}}{r_{m}}\left[1-\frac{1}{2}\left(\frac{r}{r_{m}}\right)^{b}\right] \exp \left\{\frac{1}{b}\left[1-\left(\frac{r}{r_{m}}\right)^{b}\right]\right\}$.

The governing equation is solved numerically using the second-order Adams-Bashforth scheme for time and the fourth-order differencing scheme for space. $V_{m}$ and $r_{m}$ are specified as $30 \mathrm{~m} \mathrm{~s}^{-1}$ and $120 \mathrm{~km}$, respectively. The value of $b$ is determined so that $V=15 \mathrm{~m} \mathrm{~s}^{-1}$
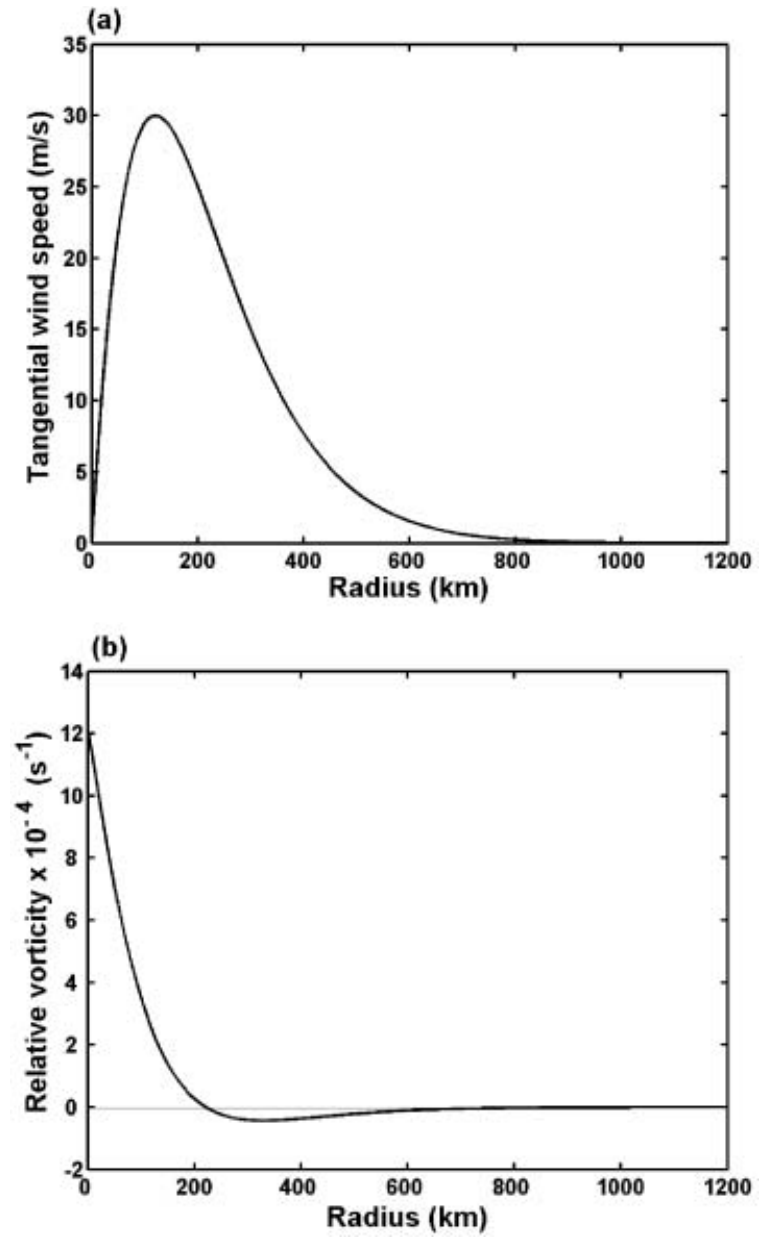

Fig. 1. Profiles of (a) tangential wind speed and (b) corresponding relative vorticity as a function of radius. These are for the control vortex with $V_{m}=30 \mathrm{~m} \mathrm{~s}^{-1}, \quad r_{m}=120 \mathrm{~km}, \quad$ and $b=1.132$ in (4).

at $r=300 \mathrm{~km}(b=1.132)$. The vortex with these parameter values will be called the control vortex. Figure 1 shows the profiles of tangential wind speed and corresponding relative vorticity as a function of radius for the control vortex. The relative vorticity is maximal at the vortex center, rapidly decreases with radius, and then becomes zero at $r=221 \mathrm{~km}$. Beyond that radius, the relative vorticity is negative with relatively small magnitudes. The minimum relative vorticity is located at $r=329 \mathrm{~km}$.

Initially two vortices with equal structure and strength are located zonally with their midpoint situated at the center of the model 
domain. The domain size is $12000 \mathrm{~km}$ by $12000 \mathrm{~km}$ and the grid interval is $40 \mathrm{~km}$ in both $x$ and $y$ directions. The model is integrated up to 144 hours with a time step of $100 \mathrm{~s}$. The background flow is not considered in this study.

\section{Critical separation distance}

When there is no background flow, a single axisymmetric vortex on an $f$-plane does not move since the tangential wind is perpendicular to the relative vorticity gradient. However, when two vortices are close enough to interact with each other, they can move on an $f$-plane. Suppose that two cyclonic axisymmetric vortices with equal structure and strength are initially located with their separation in an east-west direction. The tangential wind of the western vortex provides a northward flow at the center of the eastern vortex that is located to the east of the western vortex, thus causing the eastern vortex to move northward. Similarly, the tangential wind of the eastern vortex provides a southward flow at the center of the western vortex, thus causing the western vortex to move southward. Therefore, the two vortices are expected to rotate cyclonically around each other with a constant separation distance. To explain the motion of binary vortices, a vorticity advection process should be also taken into account. In this study, we adopt the common notion that two vortices merge (separate) if their initial separation distance is smaller (larger) than the critical separation distance. If their initial separation distance is equal to the critical separation distance, the two vortices will rotate cyclonically around each other with a constant separation distance, that is, the initial separation distance.

DeMaria and Chan (1984) suggested that the interaction of the tangential wind field of one vortex with the relative vorticity field of the other vortex adds a motion component that can result in a decrease or an increase in separation distance, depending on the sign of the relative vorticity gradient. If the center of one vortex is located in the region of negative (positive) relative vorticity gradient of the other vortex, the two vortices attract (repel) each other. According to this conception, the critical separation distance is the distance from one vortex center to the location of minimum relative vorticity. For the initial vortex profile given by (4), the critical separation distance is expressed by

$$
r_{c}=r_{m}(2+b)^{1 / b} .
$$

For the control vortex with $r_{m}=120 \mathrm{~km}$ and $b=1.132$, the critical separation distance is $329 \mathrm{~km}$. Hence, if the initial separation distance between two vortices is smaller (larger) than $329 \mathrm{~km}$, they would merge (separate) with time. However, our numerical experiments (which will be presented later) indicate that two vortices can merge with a separation distance larger than the critical separation distance proposed by DeMaria and Chan (1984). Therefore, an elaboration of DeMaria and Chan's critical separation distance is needed.

From the nondivergent barotropic model results of Pokhil et al. (1990) and Chan (1993), Falkovich et al. (1995) noted that the critical separation distance corresponds to nearly zero relative vorticity values between two vortices and thus the vortices merge when the relative vorticity values between them are positive. They explained this by the advection of the asymmetric vorticity of one vortex by the symmetric tangential flow of the other vortex. Through baroclinic model experiments, Falkovich et al. (1995) found that the presence of small positive relative vorticity values at certain levels between three-dimensional asymmetric vortices might be insufficient for their merger condition and that the values should be larger than a certain threshold value. A plausible proposal on the critical separation distance might be that binary vortices would attract (repel) each other when the initial relative vorticity between them is positive (negative). This proposal will be called Falkovich et al.'s proposal, although their result from baroclinic model experiments is stated qualitatively based upon the relative vorticity field at selected times (not at an initial time). According to this conception, the critical separation distance is twice the radius at which the relative vorticity of one vortex becomes zero. The critical separation distance corresponding to the initial vortex profile (4) is given by

$$
r_{c}=2 r_{m} 2^{1 / b} .
$$

For the control vortex, the calculated critical separation distance is $443 \mathrm{~km}$. The difference in the critical separation distance between (6) 
(a)

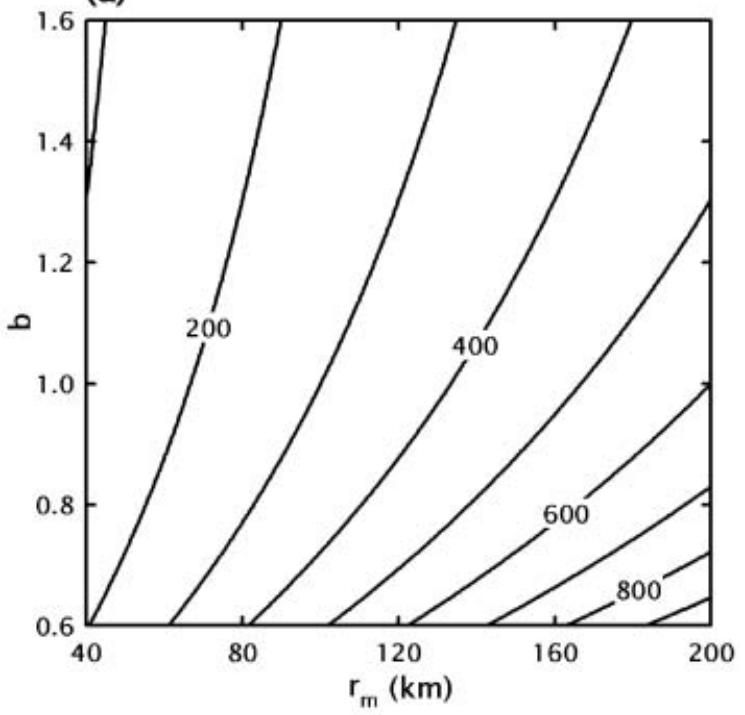

(b)

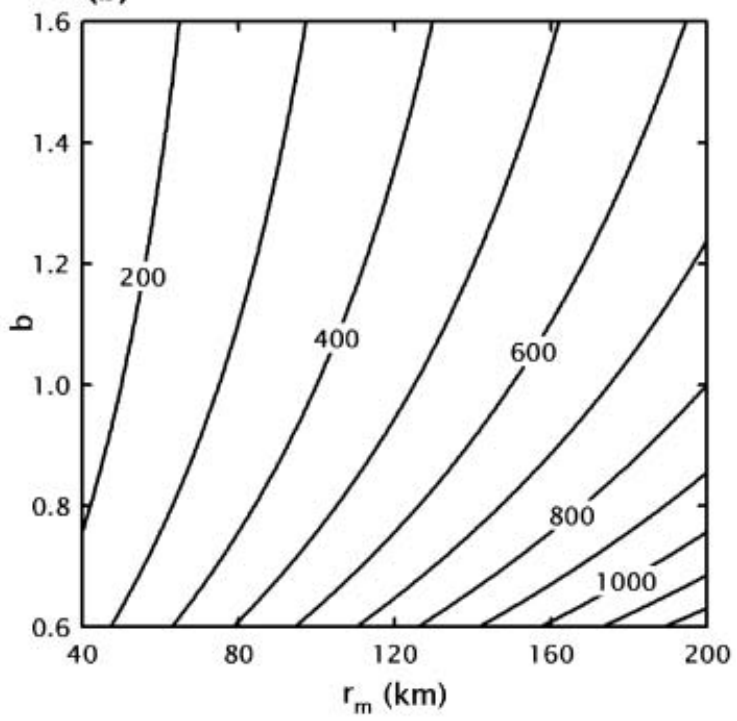

Fig. 2. Isolines of the critical separation distance $r_{c}$ as functions of $r_{m}$ and $b$. The upper figure (a) is plotted using (6) and the lower figure (b) is plotted using (7). The contour interval is $100 \mathrm{~km}$.

and (7) for the control vortex is as large as $114 \mathrm{~km}$.

In Fig. 2, the critical separation distances by DeMaria and Chan's and Falkovich et al.'s proposals are plotted as functions of $r_{m}$ and $b$. In both cases, the critical separation distance increases as $b$ decreases (that is, the vortex size increases) and the radius of maximum tangen- tial wind speed $\left(r_{m}\right)$ increases. It is noted that the critical separation distance by DeMaria and Chan's proposal is always smaller than that by Falkovich et al.'s for given $r_{m}$ and $b$.

In this study, extensive numerical experiments are performed to examine whether Falkovich et al.'s proposal on the critical separation distance is valid. Our numerical results will be presented and discussed in the next section.

\section{Motion of binary vortices on an $f$-plane}

\subsection{Control vortex pair}

To verify the relevance of Falkovich et al.'s proposal and understand the motion dynamics of binary vortices, numerous numerical experiments with different initial separation distances on an $f$-plane $[\beta=0$ in (1)] are conducted with a pair of control vortices. In this subsection, simulation results from two representative cases (merger and separation) are presented and discussed.

According to Falkovich et al.'s proposal, if the initial separation distance $(d)$ is $400 \mathrm{~km}$, the two vortices are expected to merge because

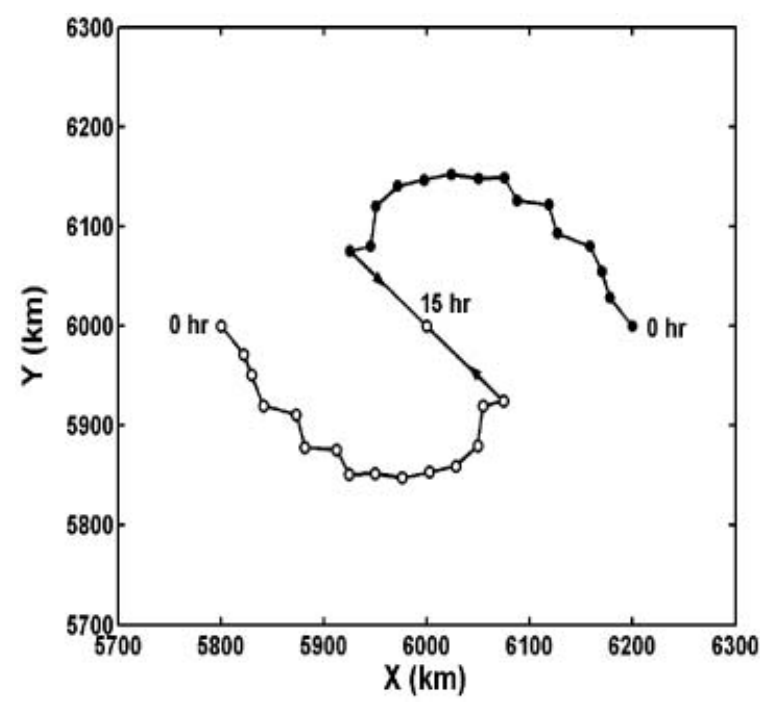

Fig. 3. Tracks of a pair of vortices in the control vortex pair experiment with $V_{m}=30 \mathrm{~m} \mathrm{~s}^{-1}, r_{m}=120 \mathrm{~km}$, and $b=1.132$ in (4). The initial separation distance between the vortices is $400 \mathrm{~km}$. The position of the center of each vortex is indicated in 1-h intervals. 

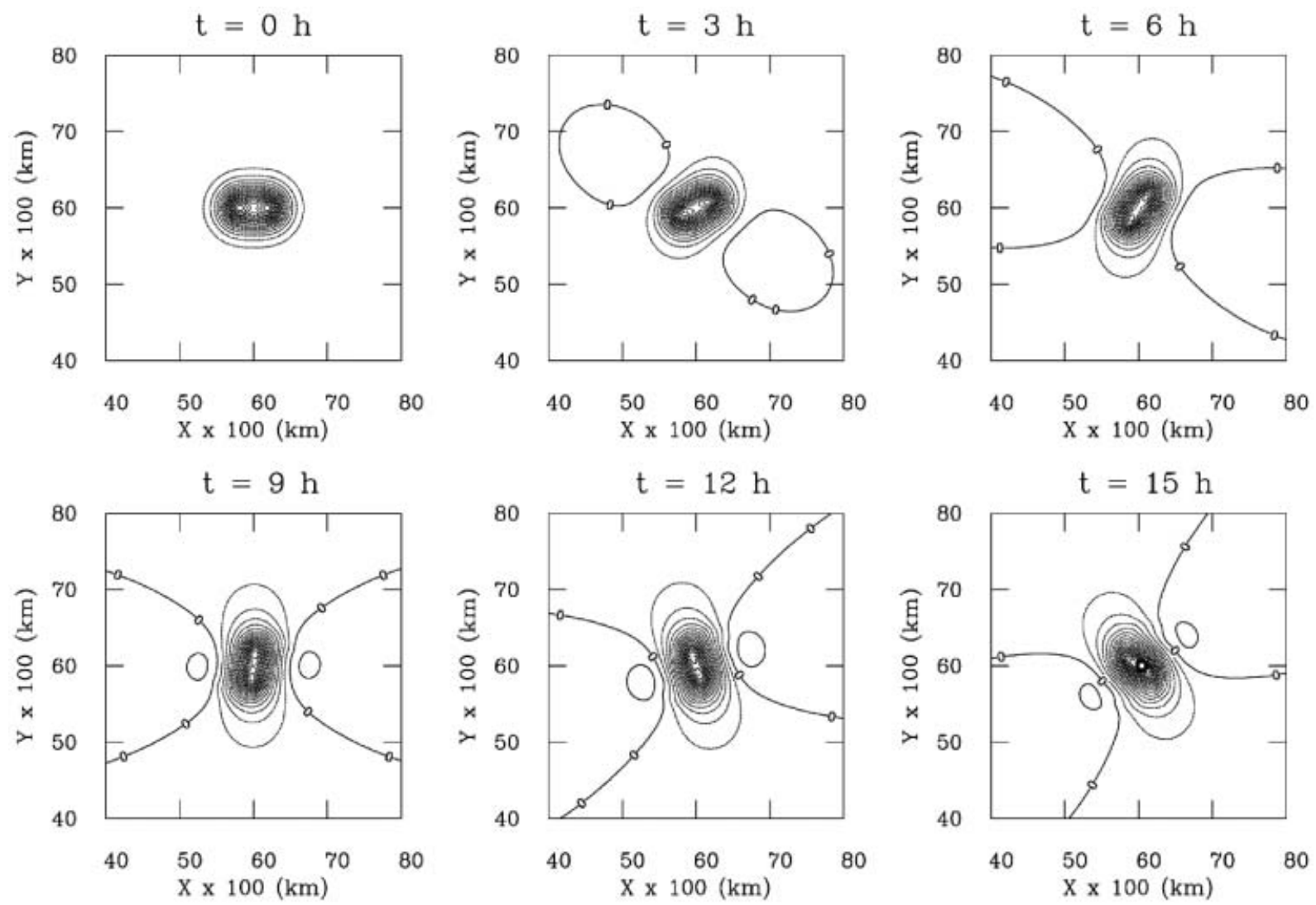

Fig. 4. Time evolution of streamfunction field in the control vortex pair experiment. The initial separation distance between the vortices is $400 \mathrm{~km}$. The contour interval is $6 \times 10^{5} \mathrm{~m}^{2} \mathrm{~s}^{-1}$.

the critical separation distance $\left(r_{c}\right)$ is $443 \mathrm{~km}$. On the other hand, separation of the two vortices is expected according to DeMaria and Chan's proposal $\left(r_{c}=329 \mathrm{~km}\right)$. Our numerical experiment (Fig. 3) shows that the two vortices rotate cyclonically with the separation distance decreasing with time. After about $14 \mathrm{~h}$, the two vortices merge. From this and other numerical experiments, we found that Falkovich et al.'s proposal is more appropriate than DeMaria and Chan's.

The time evolution of the streamfunction field in the case of $d=400 \mathrm{~km}$ is shown in Fig. 4. The center of each vortex is well identified as a streamfunction minimum. At $t=3 \mathrm{~h}$, anticyclonic flow is observed in the southeastern and northwestern regions, the formation of which is attributed to the negative relative vorticity advection. Those anticyclonic flow regions expand at $t=6 \mathrm{~h}$ and the anticyclonic flow strength- ens at $t=9 \mathrm{~h}$. As the two vortices rotate cyclonically, the anticyclonic flow regions also rotate cyclonically. The average rotational speed of each vortex before merger is $9.7 \% \mathrm{~h}$. The anticyclonic flow helps to form a steep gradient of the streamfunction (Fig. 4), which acts to increase wind speed and thus can influence the vortex movement.

Figure 5 shows the relative vorticity advection field at $t=0 \mathrm{~h}, 1 \mathrm{~h}, 2 \mathrm{~h}$, and $3 \mathrm{~h}$ in the case of $d=400 \mathrm{~km}$. At $t=0 \mathrm{~h}$, the positive relative vorticity advection is formed north (south) of the eastern (western) vortex and the negative relative vorticity advection is formed south (north) of the eastern (western) vortex. This pattern of the initial relative vorticity advection causes the eastern vortex to move northward and the western vortex southward. The initial distribution of the relative vorticity advection is gradually distorted with time by non- 

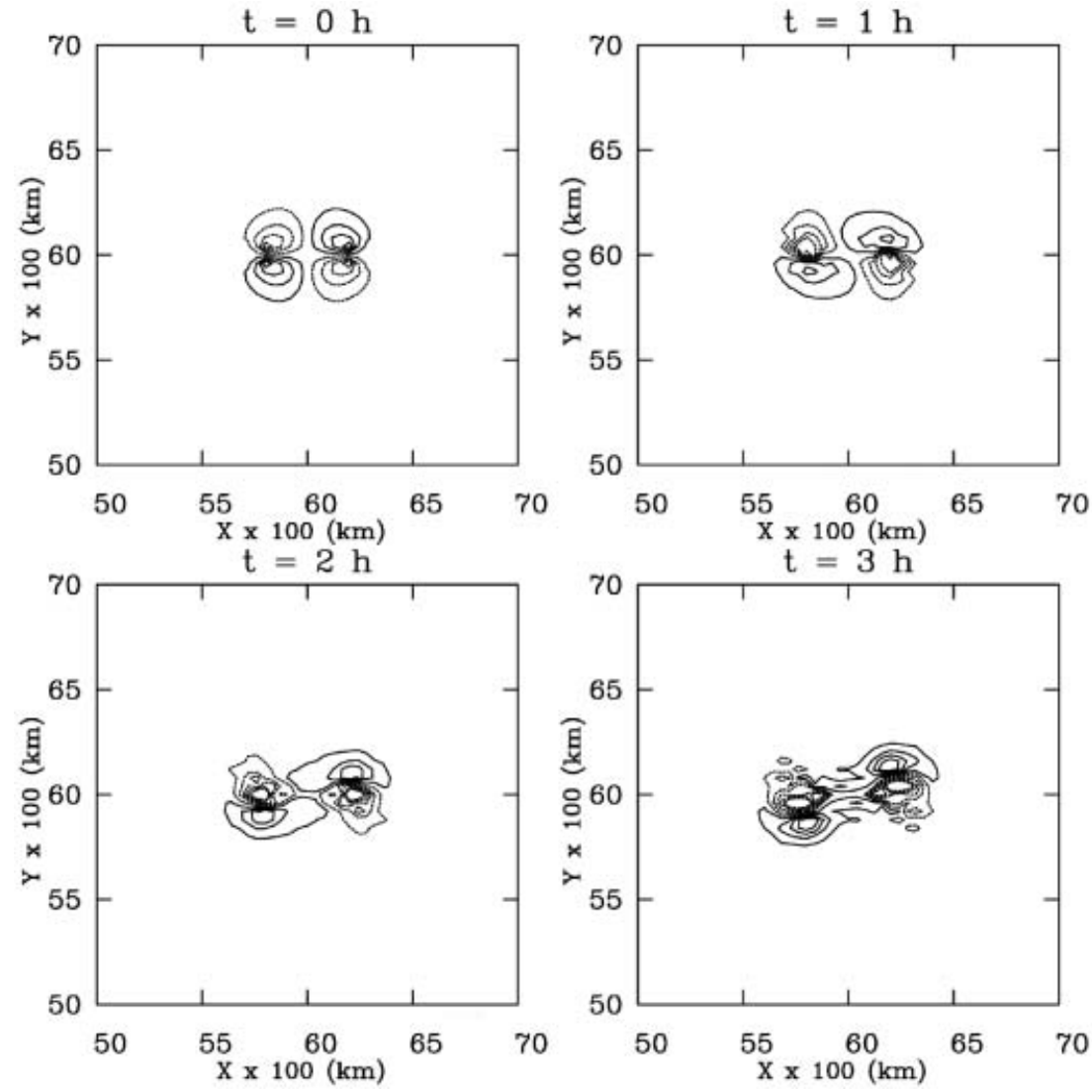

Fig. 5. Time evolution of relative vorticity advection field in the control vortex pair experiment. The initial separation distance between the vortices is $400 \mathrm{~km}$. The contour interval is $2 \times 10^{-8} \mathrm{~s}^{-1}$ and the zero contour lines are not drawn for the clarity of figure.

linear relative vorticity advection. The region between the two vortices becomes occupied with positive relative vorticity advection that strengthens with time. At $t=3 \mathrm{~h}$, the two regions of negative vorticity advection are separated by the intruding region of positive relative vorticity advection between the two vortices. An examination of the time evolution of the relative vorticity field (not shown) reveals that the relative vorticity in the region between the two vortices is positive at all times.

In the experiment with an initial separation distance of $600 \mathrm{~km}$, the separation distance between the two vortices increases with time (Fig. 6). The eastern vortex moves northnortheastward and the western vortex southsouthwestward. The movement speed of each vortex tends to decrease as time progresses. Figure 7 shows the time evolution of the streamfunction field in the case of $d=600 \mathrm{~km}$.
The separation of the two vortices is distinct in the streamfunction field. As in the merger case (Fig. 4), the gradient of the negative streamfunction is large in the regions adjacent to the anticyclonic flow. The gross patterns of relative vorticity advection at early times in the case of $d=600 \mathrm{~km}$ (Fig. 8) are similar to those for the merger case (Fig. 5). Unlike the merger case, the regions with large positive relative vorticity advection move outward with time.

\subsection{Size change}

It is well known that the motion of a single vortex on a $\beta$-plane is more sensitive to size change than to intensity change (DeMaria 1985; Chan and Williams 1987; Fiorino and Elsberry 1989). Through numerical experiments using our nondivergent barotropic vorticity equation model, we found that the motion of binary vortices on an $f$-plane is also more 


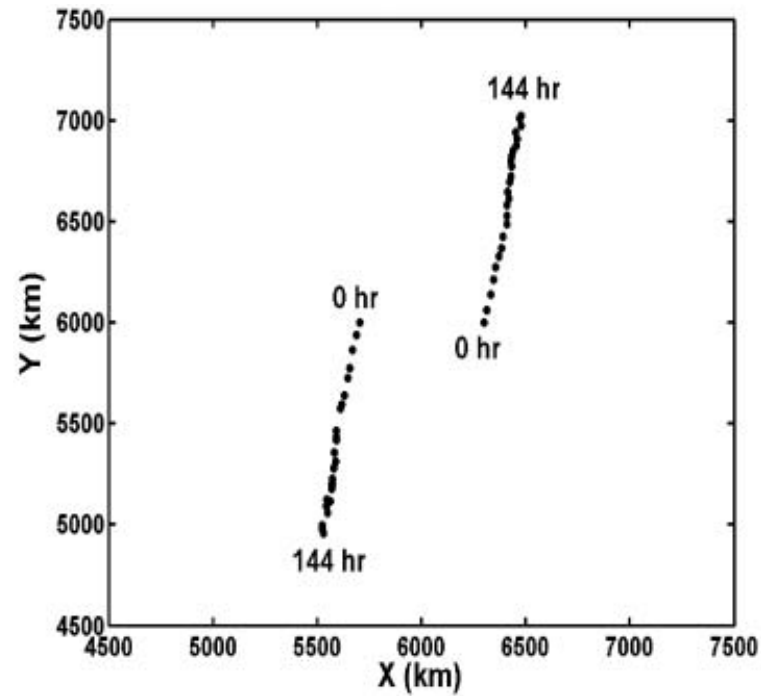

Fig. 6. Tracks of a pair of vortices in the control vortex pair experiment. The initial separation distance between the vortices is $600 \mathrm{~km}$. The position of the center of each vortex is indicated in 6 -h intervals.

sensitive to size change than to intensity change. As $b$ in (4) decreases (increases), the tangential wind in the outer region increases (decreases) and thus the vortex size increases (decreases). Variation in $b$ also changes the spatial distribution of the relative vorticity field. Notice that the critical separation distance [(6) or (7)] is independent of the maximum tangential wind speed $V_{m}$. Extensive numerical experiments with different values of $b$ are conducted to validate the critical separation distance. In this subsection, results from two experiments are presented and discussed.

Figure 9a shows the tangential wind speed as a function of radius for a vortex with $b=1.3$ in (4). Also, the control vortex with $b=1.132$ is plotted for comparison. In both the vortex profiles, $V_{m}=30 \mathrm{~m} \mathrm{~s}^{-1}$ and $r_{m}=120 \mathrm{~km}$. The tangential wind outside of the radius of maximum wind is weaker in the vortex with $b=1.3$ than in the control vortex with $b=1.132$. With this tangential wind profile and $d=400 \mathrm{~km}$, the initial relative vorticity between the two vortices is positive and the calculated critical separation distance is $409 \mathrm{~km}$ according to Falkovich et al.'s proposal. Although the merger of the two vortices is expected, the numerical ex- periment shows that the two vortices separate with time (Fig. 9b). The eastern vortex moves northwestward and the western vortex southeastward.

The difference between the critical separation distance and the initial separation distance in the experiment shown in Fig. $9 \mathrm{~b}$ is only $9 \mathrm{~km}$, but the grid interval is $40 \mathrm{~km}$. To check whether the result in Fig. 9b depends on grid interval, three additional experiments were performed with grid intervals of 20,10 , and $5 \mathrm{~km}$. Other conditions were set to be the same as those in Fig. 9b. It was found that the two vortices separate with time in all three simulations.

In the case of $b=0.7$ (Fig. 10a), the critical separation distance according to Falkovich et al.'s proposal is $646 \mathrm{~km}$ and with an initial separation distance of $600 \mathrm{~km}$ the initial relative vorticity between the two vortices is positive. The two vortices rotate cyclonically with the separation distance gradually decreasing with time and eventually merge at $t=47 \mathrm{~h}$ (Fig. 10b). Although this case (Fig. 10b) and the separation case in the control vortex pair experiment (Fig. 6) have the same initial separation distance $(d=600 \mathrm{~km})$, the change of outer tangential wind profile (size change) results in dramatically different vortex movement directions.

\subsection{Different vortex profiles}

Numerical experiments with vortex profiles different from (4) are performed to further validate the critical separation distance. For this, the initial tangential wind profile used by Chang (1983) is first employed, which is given by

$$
V(r)=B r \exp \left(-\frac{r^{2}}{r_{e}^{2}}\right),
$$

where $B$ is a constant and $r_{e}$ is the $e$-folding radius. The relative vorticity profile corresponding to (8) is

$$
\zeta(r)=2 B\left(1-\frac{r^{2}}{r_{e}^{2}}\right) \exp \left(-\frac{r^{2}}{r_{e}^{2}}\right) .
$$

According to Falkovich et al.'s proposal, the critical separation distance for the vortex profile (8) is

$$
r_{c}=2 r_{e} .
$$



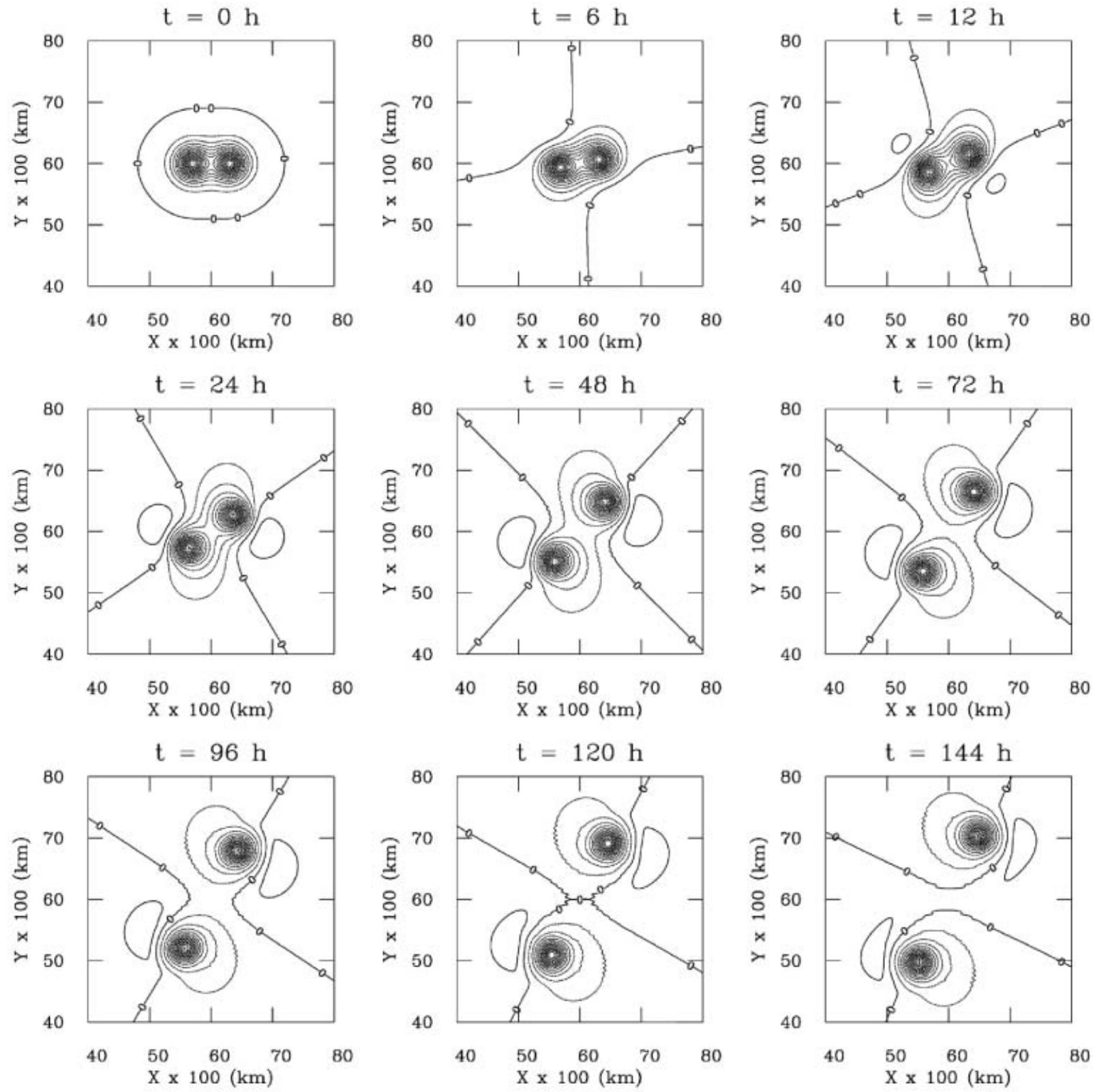

Fig. 7. Time evolution of streamfunction field in the control vortex pair experiment. The initial separation distance between the vortices is $600 \mathrm{~km}$. The contour interval is $6 \times 10^{5} \mathrm{~m}^{2} \mathrm{~s}^{-1}$.

The values of $B$ and $r_{e}$ are specified as $3.2 \times 10^{-4} \mathrm{~s}^{-1}$ and $220 \mathrm{~km}$, respectively, so that the initial tangential wind profile of this vortex is close to that of the control vortex (Fig. 11a). The corresponding relative vorticity profile is plotted in Fig. 11b. The critical separation distance is $440 \mathrm{~km}$, which is almost equal to that of the control vortex $(443 \mathrm{~km})$.

In the experiment with an initial separation distance of $400 \mathrm{~km}$, the two vortices merge (Fig. 12a). Larger positive relative vorticity between $r=76 \mathrm{~km}$ and $r=219 \mathrm{~km}$ in comparison with the control vortex (Fig. 11b) results in a shorter time period for the merger ( 6 hours vs. 15 hours). In the experiment with an initial separation distance of $600 \mathrm{~km}$, the two vortices separate with time (Fig. 12b). In comparison with the separation case in the control vortex 

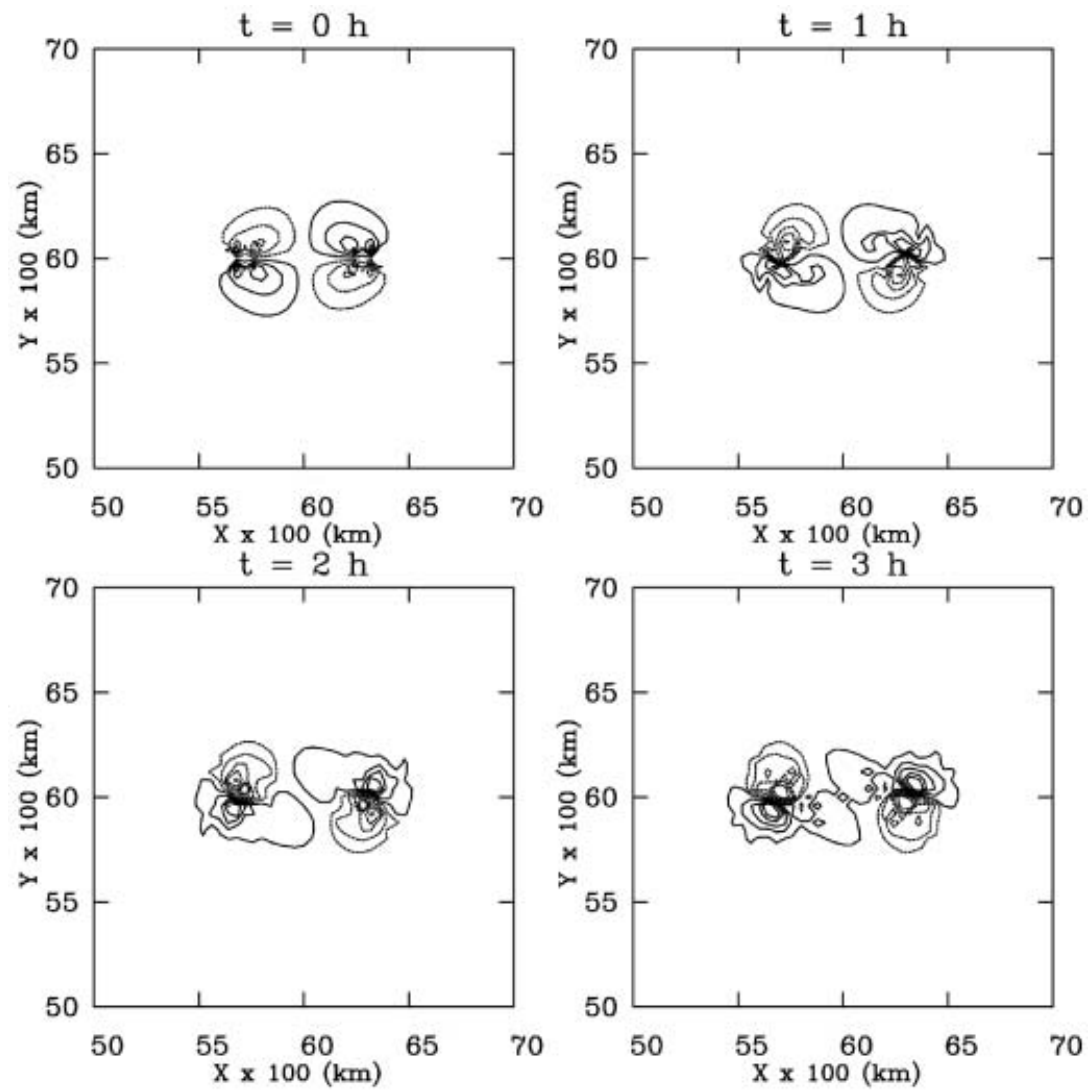

Fig. 8. Time evolution of relative vorticity advection field in the control vortex pair experiment. The initial separation distance between the vortices is $600 \mathrm{~km}$. The contour interval is $2 \times 10^{-8} \mathrm{~s}^{-1}$ and the zero contour lines are not drawn for the clarity of figure.

pair experiment with $d=600 \mathrm{~km}$ (Fig. 6), the eastern vortex is deflected more eastward and the western vortex more westward. The results presented in Figs. 12a and $12 \mathrm{~b}$ are consistent with Falkovich et al.'s proposal on the critical separation distance of binary vortices.

The initial tangential wind profile considered next is

$$
\begin{array}{ll}
V(r)=a r & \text { for } 0 \leq r \leq r_{m}, \\
V(r)=\frac{c}{r} & \text { for } r>r_{m},
\end{array}
$$

where $a$ and $c$ are constants with $c=a r_{m}^{2}$. The relative vorticity profile corresponding to (11) is given by

$$
\begin{array}{ll}
\zeta(r)=2 a & \text { for } 0 \leq r<r_{m}, \\
\zeta(r)=0 & \text { for } r>r_{m} .
\end{array}
$$

The vortex (11) is the so-called Rankine vortex for which the inner region $\left(0 \leq r \leq r_{m}\right)$ rotates like a solid body and the outer region $\left(r>r_{m}\right)$ is irrotaional. The critical separation distance according to Falkovich et al.'s proposal is

$$
r_{c}=2 r_{m}
$$

In (11), $a$ and $c$ are specified so that $V_{m}=$ $30 \mathrm{~m} \mathrm{~s}^{-1}$ at $r_{m}=120 \mathrm{~km}\left(a=2.5 \times 10^{-4} \mathrm{~s}^{-1}\right.$ and $c=3.6 \times 10^{6} \mathrm{~m}^{2} \mathrm{~s}^{-1}$ ). Then, the critical separation distance is $240 \mathrm{~km}$.

Using the vortex profile, numerical experiments are performed with initial separation distances of $320 \mathrm{~km}$ and $600 \mathrm{~km}$. In the case of $320 \mathrm{~km}$ (Fig. 13a), the two vortices attract each other and merge at $t=3 \mathrm{~h}$. This result does not follow Falkovich et al.'s proposal. For the Rankine vortex pair experiment, the relative vorticity in the inner region is uniformly large. Hence, as time progresses, the relative vorticity 
(a)

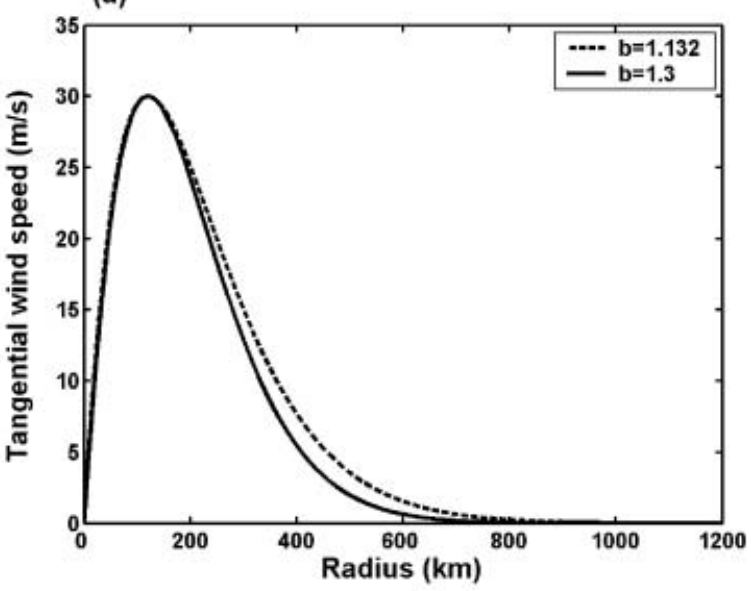

(b)

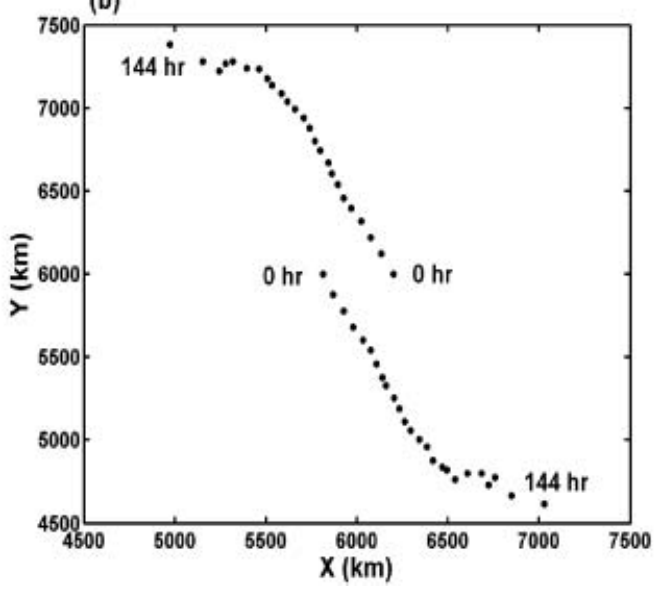

Fig. 9. (a) Profile of tangential wind speed as a function of radius and (b) tracks of a pair of vortices in the vortex pair experiment with $V_{m}=30 \mathrm{~m} \mathrm{~s}^{-1}$, $r_{m}=120 \mathrm{~km}$, and $b=1.3$ in (4). In (a), the tangential wind profile for the control vortex with $b=1.132$ is also plotted for comparison. In (b), the initial separation distance between the vortices is $400 \mathrm{~km}$ and the position of the center of each vortex is indicated in 6-hour intervals.

between the two vortices becomes positive and large by relative vorticity advection, causing the vortices to merge. In a study of the interaction of binary vortices using a discrete vortex patch model, Ritchie and Holland (1993) showed that vortices of equal size and intensity at less than critical separation (about $3.2 r_{m}$ ) merge, regardless of their initial separation distance. Through numerical experiments with (a)

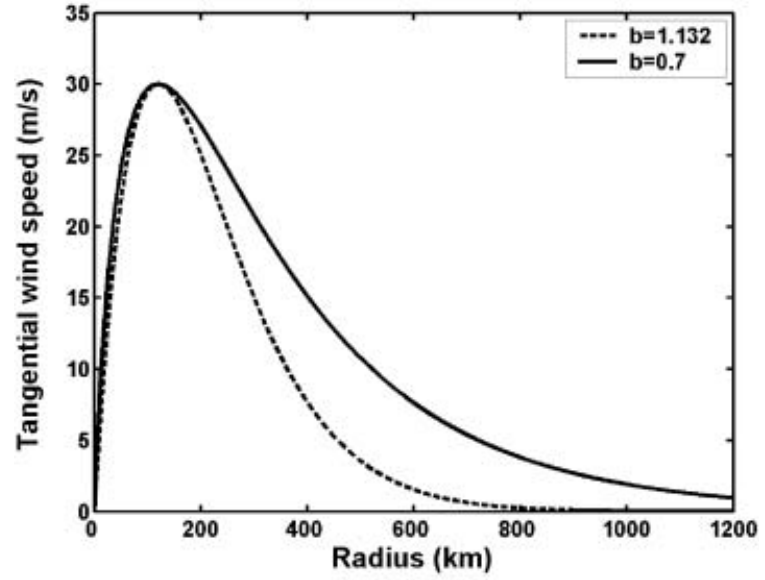

(b)

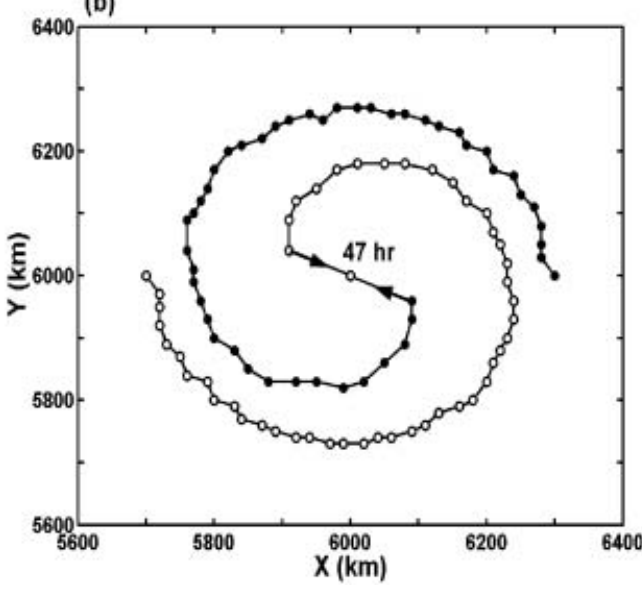

Fig. 10. (a) Profile of tangential wind speed as a function of radius and (b) tracks of a pair of vortices in the vortex pair experiment with $V_{m}=30 \mathrm{~m} \mathrm{~s}^{-1}$, $r_{m}=120 \mathrm{~km}$, and $b=0.7$ in (4). In (a), the tangential wind profile for the control vortex with $b=1.132$ is also plotted for comparison. In (b), the initial separation distance between the vortices is $600 \mathrm{~km}$ and the position of the center of each vortex is indicated in 1-hour intervals.

the Rankine vortex pair, we found that two vortices merge if the initial separation distance is less than $3.3 r_{m}$. Our result is consistent with Ritchie and Holland's. In the case of $d=600 \mathrm{~km}$ (Fig. 13b), the two vortices rotate cyclonically without merger/separation. We found that when the initial separation distance is larger than about $400 \mathrm{~km}\left(3.3 r_{m}\right)$, two vortices rotate cyclonically without merger/ 

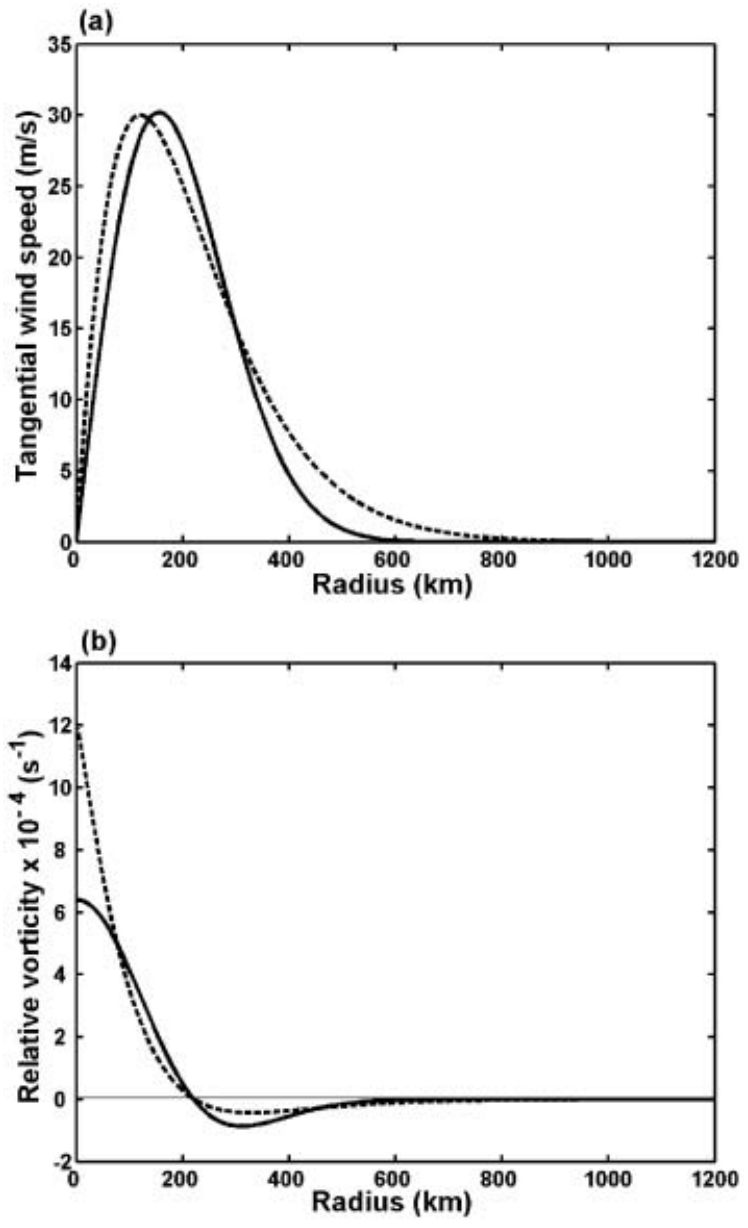

Fig. 11. Profiles of (a) tangential wind speed and (b) corresponding relative vorticity as a function of radius for a vortex with $B=3.2 \times 10^{-4} \mathrm{~s}^{-1}$ and $r_{e}=220 \mathrm{~km}$ in (8). The profiles of tangential wind speed and corresponding relative vorticity for the control vortex (dashed lines) are also plotted for comparison.

separation. This result is also consistent with Ritchie and Holland's.

The above numerical results indicate that the critical separation distance proposed by Falkovich et al. (1995) cannot be applied to the Rankine vortex pair. Since the vortices we are concerned with in this study are binary tropical cyclones and the relative vorticity in tropical cyclones exhibits a maximum at the vortex center and typically decreases rapidly with radius, we can comfortably exclude the (a)

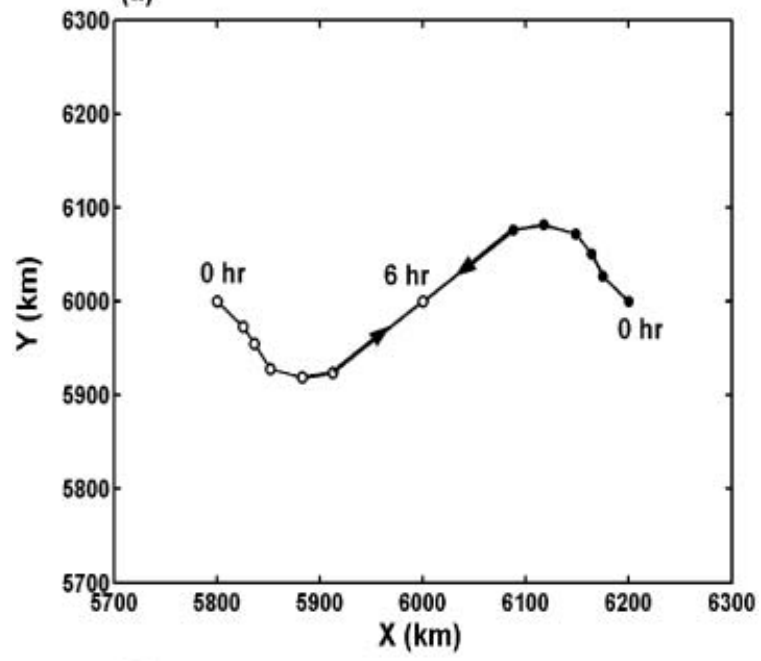

(b)

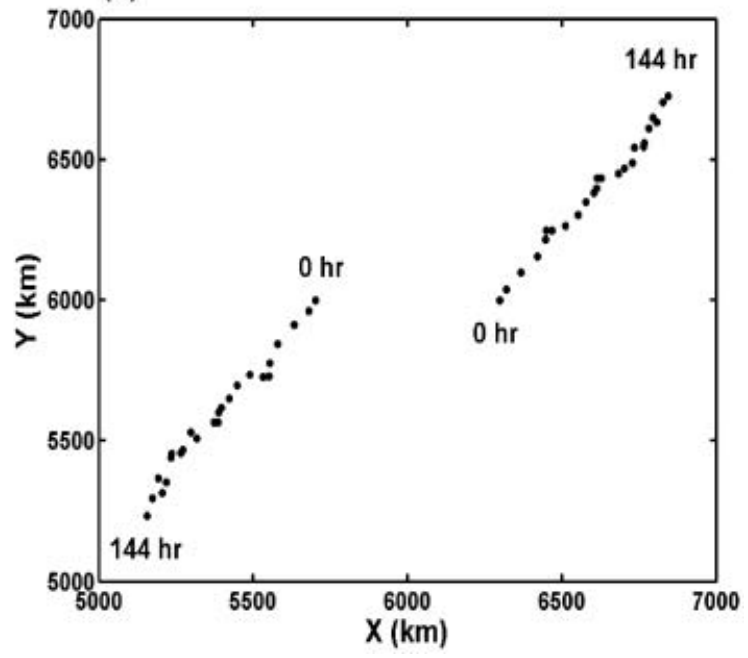

Fig. 12. Tracks of a pair of vortices in the vortex pair experiments with $B=3.2 \times 10^{-4} \mathrm{~s}^{-1}$ and $r_{e}=220 \mathrm{~km}$ in (8). The initial separation distance between the vortices is $400 \mathrm{~km}$ in (a) and $600 \mathrm{~km}$ in (b). The position of the center of each vortex is indicated in 1-h intervals in (a) and in 6-h intervals in (b).

Rankine vortex case in critical separation distance arguments.

\subsection{Proposal and conceptual explanation}

In all tested cases that have initially negative relative vorticity in the mid-region between two vortices, it is found that the two vortices separate with time. However, it is found that every case that has initially positive relative 
(a)

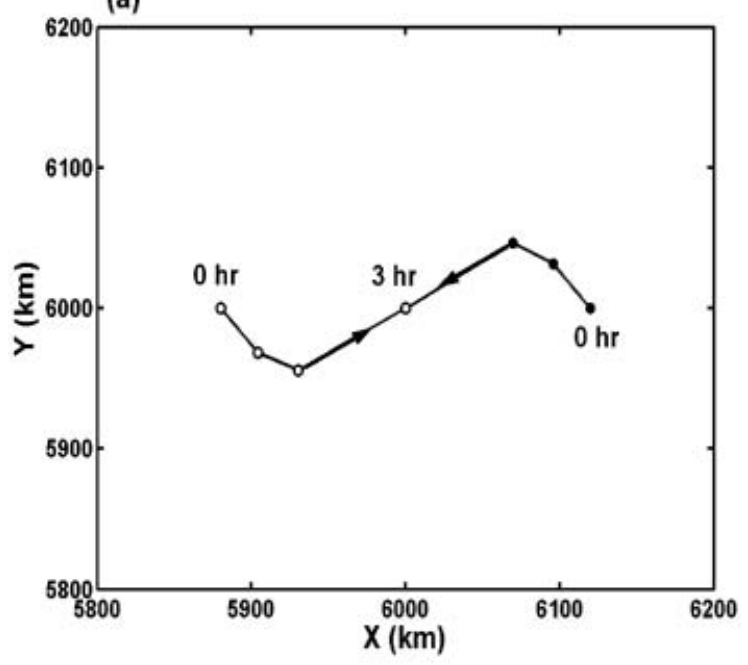

(b)

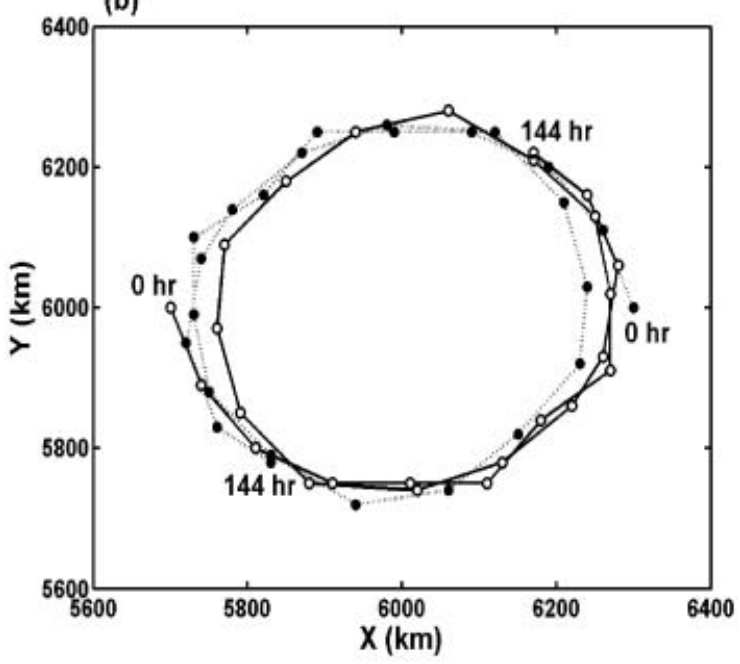

Fig. 13. Tracks of a pair of vortices in the Rankine vortex pair experiments with $a=2.5 \times 10^{-4} \mathrm{~s}^{-1}$ and $c=3.6 \times 10^{6} \mathrm{~m}^{2} \mathrm{~s}^{-1}$ in (11). The initial separation distance between the vortices is $320 \mathrm{~km}$ in (a) and $600 \mathrm{~km}$ in (b). The position of the center of each vortex is indicated in 1 -h intervals in (a) and in 6-h intervals in (b).

vorticity in the mid-region between two vortices does not guarantee their merger. When the initial relative vorticity in the mid-region between two vortices is small enough, the two vortices separate. This is the case shown in Fig. 9 in which the critical separation distance is slightly larger than the initial separation distance. In this case, the initial maximum relative vorticity in the mid-region between the two vortices is $1.39 \times 10^{-5} \mathrm{~s}^{-1}$. For a given initial vortex profile, only when the initial positive relative vorticity in the mid-region between two vortices is larger than a certain threshold value, they merge.

Based upon the careful analyses of numerous numerical experiments, we found that the actual critical separation distance is slightly smaller than that by Falkovich et al.'s proposal. Thus, for the vortex profile (4), our proposed critical separation distance, in a modification to Falkovich et al.'s proposal, takes the form

$$
r_{c}^{\prime}=2\left(r_{m} 2^{1 / b}-\alpha\right) \text {. }
$$

The constant $\alpha$ can be determined from the experiment for which the vortex pair rotates circularly with nearly the same separation distance, that is, the experiment of the initial separation distance being equal to the critical separation distance. To find this case for a given vortex profile, numerical experiments are performed with different initial separation distances. It is determined that $\alpha=15 \mathrm{~km}$ for the vortex profile (4) with $V_{m}=30 \mathrm{~m} \mathrm{~s}^{-1}$, $r_{m}=120 \mathrm{~km}$, and $b=1.132$ (control vortex, see subsection 4.1 ), $\alpha=16 \mathrm{~km}$ for the vortex profile (4) with $V_{m}=30 \mathrm{~m} \mathrm{~s}^{-1}, r_{m}=120 \mathrm{~km}$, and $b=1.3$ (see subsection 4.2 ), and $\alpha=7 \mathrm{~km}$ for the vortex profile (4) with $V_{m}=30 \mathrm{~m} \mathrm{~s}^{-1}$, $r_{m}=120 \mathrm{~km}$, and $b=0.7$ (see subsection 4.2 ). Our proposed critical separation distance for the vortex profile (8) is

$$
r_{c}^{\prime}=2\left(r_{e}-\alpha\right)
$$

The constant $\alpha$ is determined to be $18 \mathrm{~km}$ for the vortex profile (8) with $B=3.2 \times 10^{-4} \mathrm{~s}^{-1}$ and $r_{e}=220 \mathrm{~km}$. The value of $\alpha$ in (14) [(15)] is much smaller than $r_{m} 2^{1 / b}\left[r_{e}\right]$. To check whether $\alpha$ is dependent upon grid interval, numerical experiments are performed with grid intervals of 20 and $10 \mathrm{~km}$ for the control vortex pair. Results showed that $\alpha$ in the finer model resolution experiments is nearly the same as that in the $40 \mathrm{~km}$ model resolution experiment.

As described in subsection 4.2, the result of the numerical experiment using the vortex profile (4) with $V_{m}=30 \mathrm{~m} \mathrm{~s}^{-1}, r_{m}=120 \mathrm{~km}$, and $b=1.3$ for the case of an initial separation distance of $400 \mathrm{~km}$ (Fig. 9) deviates from Falkovich et al.'s proposal that the two vortices 
merge because $d(400 \mathrm{~km})<r_{c}(409 \mathrm{~km})$. Actually, the two vortices separate with time. In addition, this case does not follow the notion that two vortices merge if $d<r_{c}$. However, according to the modified proposal, the critical separation distance for this case is $377 \mathrm{~km}$, thus $d$ $(400 \mathrm{~km})>r_{c}^{\prime}(377 \mathrm{~km})$, suggesting separation of the two vortices. The numerical result in Fig. 9 supports our proposal. Also, with our proposal, this case follows the notion that two vortices separate if $d>r_{c}^{\prime}$. It should be noted that the constant $\alpha$ cannot be determined a priori and thus the proposed expression of the critical separation distance may not be directly applied in the forecasting problem.

The merger or separation of a pair of vortices can be conceptually explained with schematics at $t=0 \mathrm{~h}$, as depicted in Fig. 14. Here, the advection of the (symmetric) relative vorticity of one vortex by the symmetric tangential wind of the other vortex is taken into account. In Fig. 14, the relative vorticity of the western vortex and the symmetric tangential wind vector of the eastern vortex are drawn. Let us first explain a mechanism leading to the merger of two vortices (Fig. 14c). This is a case with positive relative vorticity exceeding a threshold value in the mid-region between the two vortices (Fig. 3, Fig. 10, Fig. 12a). To the north of the center of the eastern vortex, positive vorticity anomaly is produced due to the advection of the relative vorticity of the western vortex by the symmetric tangential wind of the eastern vortex. (The meaning of positive vorticiy anomaly here is the positive tendency of relative vorticity due to the positive vorticity advection.) To the south of the center of the eastern vortex positive vorticity anomaly is also produced due to the advection of the relative vorticity of the western vortex by the symmetric tangential wind of the eastern vortex. For the merger case, the difference between relative vorticity in the western periphery of the eastern vortex and relative vorticity in its southern periphery is larger than that between relative vorticity in the eastern periphery of the eastern vortex and relative vorticity in its northern periphery. Therefore, positive vorticity anomaly is larger to the south of the center of the eastern vortex than to the north of the center of the eastern vortex. Accordingly, the net secondary flow near the center of the eastern vortex is west- ward. Similarly, the net eastward secondary flow is formed near the center of the western vortex. This causes the two vortices to attract each other. This explanation is essentially the same as that of Falkovich et al. (1995).

Figure 14a depicts a case with negative relative vorticity in the mid-region between two vortices (Fig. 6, Fig. 12b). In this case, positive vorticity anomaly is developed to the north of the center of the eastern vortex and negative vorticity anomaly is developed to the south. Both the vorticity anomalies make secondary flows near the center of the eastern vortex toward the east. Similarly, both vorticity anomalies to the north and south of the western vortex induce secondary flows near its center that are directed westward. Hence, the two vortices repel each other.

Figure $14 \mathrm{~b}$ depicts a case in which relative vorticity in the mid-region between the two vortices is positive, but its value is smaller than a threshold value for their merger (Fig. 9). In this case, positive vorticity anomaly is produced to the north of the eastern vortex. To the south of the eastern vortex, positive voticity anomaly, the magnitude of which is smaller than that of positive vorticity anomaly to the north of the center of the eastern vortex, is produced. The net secondary flow near the center of the eastern vortex is eastward. Similarly, the net westward secondary flow is formed near the center of the western vortex. As in the case of Fig. $14 \mathrm{a}$, the two vortices repel each other. Our explanation for the separation cases (Fig. 14a and Fig. 14b) extends that for the merger case (Fig. 14c) by Falkovich et al. (1995).

The above conceptual explanation is based upon the advection of the relative vorticity of one vortex by the symmetric tangential wind of the other vortex. In this regard, the explanation is similar to that of DeMaria and Chan (1984). However, our explanation is different from theirs in that the magnitude of vorticity anomaly produced to the north of one vortex differs from that of vorticity anomaly produced to its south, resulting in net eastward or westward secondary flow near the vortex center. In DeMaria and Chan (1984), the vorticity anomaly to the north of one vortex has, to our understanding, the same magnitude as that to its south and its signs are different. Judging from the results from the nondivergent barotropic 
(a)

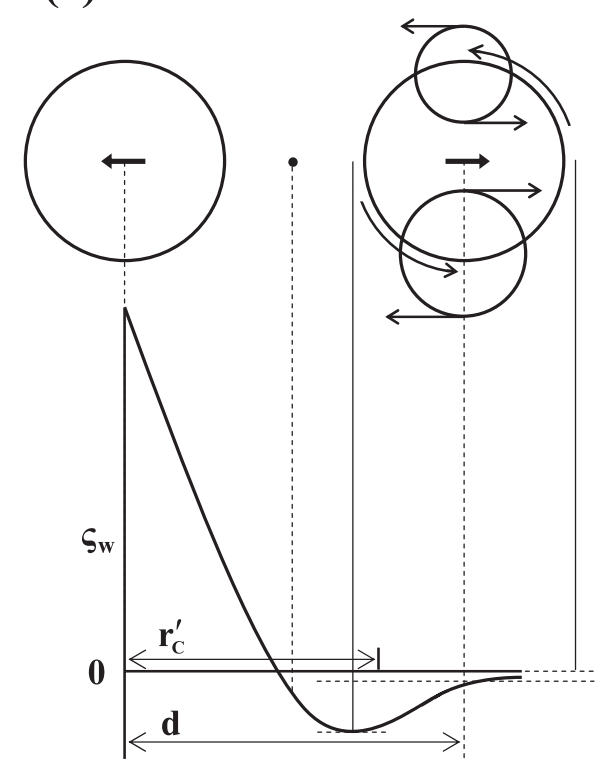

(b)

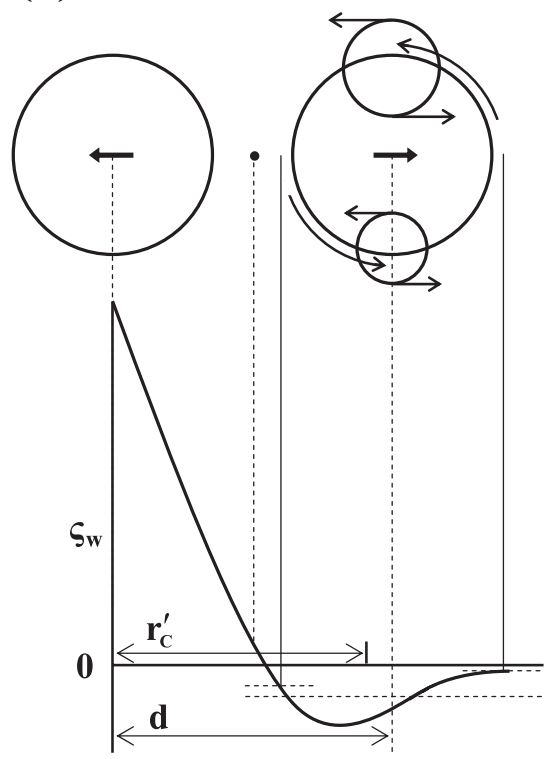

(c)

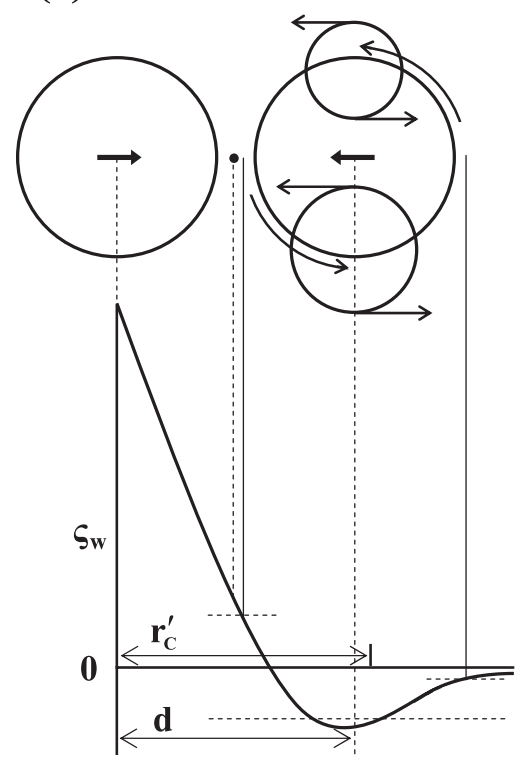

Fig. 14. Schematic illustration explaining a mechanism for the merger or separation of a pair of initial vortices: (a) a case with negative relative vorticity in the mid-region between vortices, (b) a case with positive relative vorticity smaller than a threshold value in the mid-region between vortices, and (c) a case with positive relative vorticity exceeding a threshold value in the midregion between vortices. The mid-point between the two vortices is indicated by dot circle. $\zeta_{w}$ is the relative vorticity of the western vortex, $d$ is the initial separation distance, and $r_{c}^{\prime}$ is the critical separation distance. Detailed explanations are given in the text. The schematic (c) is based upon Falkovich et al. (1995). 
model simulations, our explanation improves upon DeMaria and Chan's.

Chan and Law (1995) also explained the interaction of binary vortices with the relative vorticity advection. In their study, the asymmetric component at any time means the rest part after the symmetric component of only one vortex is removed. This rest part includes the symmetric component of the other vortex. They found that the interaction between binary vortices results from the advection of symmetric vorticity by the asymmetric flow and the advection of asymmetric vorticity by the symmetric flow. Our partition approach, also DeMaria and Chan's and Falkovich et al.'s, should be clearly distinguished from Chan and Law's in that this study considers the interaction of the symmetric relative vorticity of one vortex and the symmetric tangential wind of the other vortex. It is noticed in Chan and Law (1995) that although they did not propose a conceptual model explaining the merger or separation of binary vortices, they could present the meaningful results by the partition.

\section{Summary and conclusions}

This study investigated the critical separation distance of binary cyclonic vortices using a nondivergent barotropic model. The vortices we are concerned with here are binary tropical cyclones. Consistent with the proposal of Falkovich et al. (1995), our experimental results on an $f$-plane indicate that the sign of the initial relative vorticity in the mid-region between two vortices largely determines their merger or separation. It was shown that two vortices separate when the initial relative vorticity in the mid-region between them is negative, but an initial positive relative vorticity between the two vortices does not guarantee their merger. It appears that the two vortices merge only when the initial positive relative vorticity is larger than a certain threshold value. It was suggested that the critical separation distance of binary vortices is slightly smaller than twice the radius at which the relative vorticity of one vortex becomes zero. The merger or separation of binary vortices are conceptually explained by the advection of the symmetric relative vorticity of one vortex by the symmetric tangential flow of the other vortex. This produces vorticity anomalies with different magnitudes to the north and south of each vortex and results in net eastward or westward secondary flow near its center.

In this study, we investigated the motion of binary vortices with a focus on the condition determining their merger or separation using the simplest dynamical model that enables us to examine vortex motion, that is, using a nondivergent barotropic model on an $f$-plane. The divergent flow in a divergent barotropic model or a baroclinic model can affect the interaction of binary vortices (Chang 1983). The baroclinic effect might decrease the critical separation distance (Falkovich et al. 1995). The motion of binary vortices on an $f$-plane can be different from that on a sphere, primarily in that the vorticity centroid of the vortex pair is fixed on an $f$-plane, but can drift considerably poleward and westward on a sphere (Prieto et al. 2003). Therefore, it would be interesting to examine to what extent and how each of divergent flow, baroclinicity, and spherical geometry can modify the critical separation distance of binary vortices suggested for a nondivergent barotropic atmosphere on an $f$-plane. This deserves further investigation.

\section{Acknowledgments}

The authors would like to thank two anonymous reviewers for providing valuable comments on the original manuscript. This work was partly funded by the Korea Meteorological Administration Research and Development Program under Grant CATER 2006-2202.

\section{References}

Brand, S., 1970: Interaction of binary tropical cyclones of the western North Pacific Ocean. J. Appl. Meteor., 9, 433-441.

Chan, J.C.L., 1993: The interaction of binary vortices in a barotropic model. Tropical Cyclone Disasters, J. Lighthill, Z. Zhemin, G.J. Holland, and K.A. Emanuel, Eds., Peking University Press, 296-302.

1995: Performance of global and regional NWP models in their prediction of typhoon Nat (1991). Wea. Forecasing, 10, 400-410.

- and R.T. Williams, 1987: Analytical and numerical studies of the beta-effect in tropical cyclone motion. Part I: Zero mean flow. J. Atmos. Sci., 44, 1257-1264.

and H. Lam, 1989: Performance of the ECMWF model in predicting the movement of 
typhoon Wayne (1986). Wea. Forecasting, 4, 234-245.

and A.C.K. Law, 1995: The interaction of binary vortices in a barotropic model. Meteor. Atmos. Phys., 56, 135-155.

Chang, S.W., 1983: A numerical study of the interactions between two tropical cyclones. Mon. Wea. Rev., 111, 1806-1817.

DeMaria, M., 1985: Tropical cyclone motion in a nondivergent barotropic model. Mon. Wea. Rev., 113, 1119-1210. - and J.C.L. Chan, 1984: Comments on "A numerical study of the interactions between two tropical cyclones". Mon. Wea. Rev., 112, 16431645.

Dong, K. and C.J. Neumann, 1983: On the relative motion of binary tropical cyclones. Mon. Wea. Rev., 111, 945-953.

Falkovich, A.I., A.P. Khan, and I. Ginis, 1995: Motion and evolution of binary tropical cyclones in a coupled atmosphere-ocean numerical model. Mon. Wea. Rev., 123, 1345-1363.

Fiorino, M. and R.L. Elsberry, 1989: Some aspects of vortex structure related to tropical cyclone motion. J. Atmos. Sci., 46, 975-990.

Fujiwhara, S., 1921: The natural tendency towards symmetry of motion and its application as a principle of motion. Quart. J. Roy. Meteor. Soc., 47, 287-293.

, 1923: On the growth and decay of vortical systems. Quart. J. Roy. Meteor. Soc., 49, 75104.

, 1931: Short note on the behavior of two vor- tices. Proc. Physico-Mathematical Society Japan, 13, 3rd ser., 106-110.

Hart, R.E. and J.L. Evans, 1999: Simulations of dual-vortex interaction within environmental shear. J. Atmos. Sci., 56, 3605-3621.

Haurwitz, B., 1951: The motion of binary tropical cyclones. Arch. Meteor. Geophys. Bioklimatol., A4, 73-86.

Holland, G.J. and G.S. Dietachmayer, 1993: On the interaction of tropical-cyclone-scale vortices. III: Continuous barotropical vortices. Quart. J. Roy. Meteor. Soc., 119, 1381-1398.

Kuo, H.-C., G.T.-J. Chen, and C.-H. Lin, 2000: Merger of tropical cyclones Zeb and Alex. Mon. Wea. Rev., 128, 2967-2975.

Lander, M.A., 1995: The merger of two tropical cyclones. Mon. Wea. Rev., 123, 2260-2265.

Melander, M.V., N.J. Zabusky, and J.C. McWilliams, 1988: Symmetric vortex merger in two dimensions: Cause and condition. J. Fluid Mech., 195, 303-340.

Pokhil, A.E., I.G. Sitnikov, V.A. Zlenko, and I.V. Polyakova, 1990: Numerical experiments on investigation of atmospheric vortices. Meteor. Gidrol., 4, 21-28.

Prieto, R., B.D. McNoldy, S.R. Fulton, and W.H. Schubert, 2003: A classification of binary tropical cyclone-like vortex interactions. Mon. Wea. Rev., 131, 2656-2666.

Ritchie, E.A. and G.J. Holland, 1993: On the interaction of tropical-cyclone-scale vortices. II: Discrete vortex patches. Quart. J. Roy. Meteor. Soc., 119, 1363-1379. 